\title{
Principle Analysis of Computer Vision and Its Application Research
}

\author{
Xu Ziang \\ Newcastle University, UK NE1 7RU
}

Keywords: computer vision; robot navigation; object recognition

\begin{abstract}
This paper briefly summarizes the principles of computer vision, clarifies the current status and flow of visual principles, and enumerates several applications of computer vision in detail. It has made outstanding contributions in dealing with face testing and identification, robot orientation and navigation applications, and finally pointed out the development trend of computer vision technology.
\end{abstract}

\section{Introduction}

Due to the innovation of computer technology, computer vision has become a new type of subject, which brings together all the situational information and processing methods in the two-dimensional picture. In the process of industrial and military experiments, many new topics have been listed, such as the development of intelligent robots, new weapons, etc., and people pay more and more attention to computer vision. From 1970 to 1979, when scientists explored basic theories, they also focused on application systems. At present, computer vision theory is widely used in various fields of the world, artificial intelligence, image processing and neurobiology. In addition, computer vision is an edge discipline that combines several subjects, and its scientific research results are numerous.

\section{Computer vision overview and basic framework}

The human binocular perception system helps them to clearly see the three-dimensional surrounding scene. In the process of observing green plants, we can first see the change of leaf color and accurately detect the growth of green plants. When admiring portraits, you can quickly identify which character it is, and also express its emotional activity from the facial expression in the picture. From this, we can see that the human visual system has a certain uniqueness, can feel the real three-dimensional scene, so that researchers use sensors, computer hardware to imitate the real human vision system, to achieve situational reproduction, such as collecting, exploring, solving and learning three-dimensional environment images, add this skill to your computer, enable the computer and robot system to have intelligent visual effect. Image acquisition, image processing, image analysis and image display output are important parts of computer vision system. Therefore, the theoretical framework of computer vision includes image knowledge acquisition layer, image feature depiction layer, image data processing layer, as detailed in Figure 1.

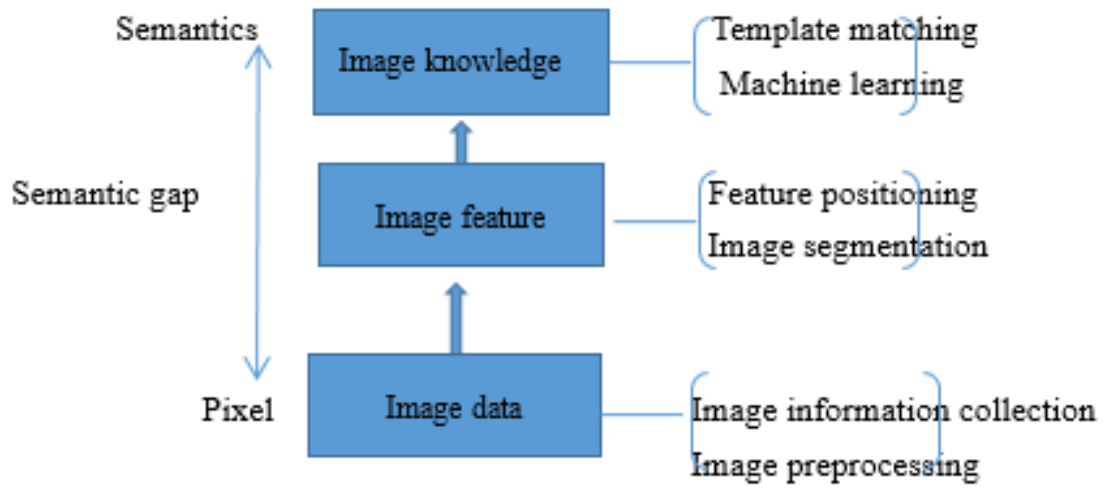

Figure 1 Computer vision system structure 


\subsection{Image data processing layer}

The main function of the image data processing layer is to operate on specified processing objects, such as pixel-type digital signals, image acquisition, decompression, conversion, transmission, saving, recovery, and the like. Image data processing layer is to change the original image into a certain characteristics of the image, such as signal ratio, and changes only the graph, which does not help the structure discussion, but it is the basic condition of the boundary detection. This technology is very mature and has a long service life, and is often used frequently in digital filtering and conversion.

Enhance the image. The main function is to enhance the visual effect of the image, to display the overall contour and special part of the desired image, to change the sharpness of the image, to broaden the difference between the features of each object, and to control the unused features, enhanced image quality, ample amount of information, improved image reading power and ability to distinguish effects, thus meeting special requirements.

Smooth the image. The main function is to highlight the largest part of the image, the core area, low frequency components, control image noise, interference with high frequency components, etc. The purpose of this is to gradually smooth the image brightness and reduce the level of mutant ladder, this solution can be more good to improve image quality.

Image data encoding and transmission. Image coding is a science technique that exhibits an existing pixel matrix to some extent with a small amount of data. Digital images involve a very large amount of data, such as pixel digital images, each pixel has $256 \mathrm{k}$ bytes, and if it is transmitted straight, it will lose time. Therefore, when digital images are converted, encoded, and compressed, and to facilitate save and transfer images.

\subsection{Image feature drawing layer}

First, the edges are sharpened. The main purpose is to make the image peripheral lines, edges, and image details more clear. The main factor of clear image blurring is because the image is affected by averaging and integral calculation, which can complete the reverse calculation and enhance the sharpness of the image. It is the basic problem of the original visual theory and algorithm, and it is also the core factor affecting the visual algorithm at the end.

Second, image segmentation. Divide the image into several designated lots with certain special performance, and explore the valuable target objects and processes by combining spatial features, spectral features, grayscale values, colors and textures. Commonly used image segmentation methods include lot segmentation method, numerical segmentation method, theoretical segmentation method and edge segmentation method. In the the 1990s, many famous scholars at home and abroad have innovated the existing segmentation method and explored a series of new segmentation methods according to the theoretical method of related disciplines. Lots that are identified and obtained with the target image are often used frequently in medical picture disorders, image language recognition, and image search.

\subsection{Image knowledge acquisition layer}

Image recognition refers to the use computers to solve, research, and understand images, so as to identify various forms of target and object technology. It is one of the key contents of computer vision system, and the content involved in image recognition includes image matching and mechanical learning.

There are many famous scholars in China who have discussed the image matching work in detail and achieved excellent results. Image matching content includes: feature range, similarity measure, and search method. First, the range of features is grayscale, feature points, edge strength, binary edges, curves, surfaces, statistical properties, models, and high-level matching. Secondly, the similarity measure has correlation coefficient, normalized correlation coefficient NCC, normalized correlation coefficient and matching filter, statistical correlation and filter, sum of absolute 
difference SSD, mask correlation, Hausdorff distance, minimum distance classifier, mutual information. Finally, search methods have hierarchical, hierarchical iterative or simulated annealing, simulated annealing, genetic algorithms, neural networks, tree or graph matching, relaxation algorithms, iterative point matching, energy minimization, and fast FFT.

In the middle of the 90, the concept of machine learning was introduced, and machine learning belongs to an artificial intelligence science, which focuses on artificial intelligence, especially the instinct to adjust scientific algorithms in the process of empirical learning. In artificial intelligence, machine learning is an important technology, and it is the core path to complete a computer with intelligent methods. It is widely used in the scope of artificial intelligence.

\section{Computer vision application}

\subsection{Face testing and identification.}

Development research status, In 1900, Francis Galton, a famous British scientist. He was the first scientist to analyze face testing and identification. In 1990-1999, face testing and identification was officially launched and developed in an independent form. At present, there is a database of oriental face images in China, which is a large and comprehensive database in the world. Face testing and identification have gone through three stages of development.

First, further analyze and discuss the facial features used in face testing and identification, and the specific identification process is guided by relevant staff.

Secondly, the human-computer interactive identification stage uses the multi-dimensional feature vector to show the facial features. In general, HAR-MON and LESK are the main representatives. In addition, the statistical identification can be expressed by KOBAVASHI and KAYA, and the European distance is incorporated into the face feature presentation.

Finally, the machine automatically identified. With the rapid development of science and technology, the face identification technology is gradually moving towards practical use, such as the face identification system launched by Eyematic. In addition, Tsinghua University's "Xv" research face identification system has been certified.

Face test and identification algorithm, The face testing and identification system uses cameras and digital cameras in the computer to gain insight into the face, thereby gaining valuable features from the influence to discriminate identity information skills. Face testing, identification, and feature acquisition are the core content of face testing and identification. The first stage is the image set, followed by face detection, and then test the amplitude of the image in the set, once a person's face appears, it is necessary to quickly determine its location. At the same time, the feature acquisition is used to complete the face identification work, thereby extracting the face data, and the final step is to identify the result.

\subsection{The function of robot target location in computer vision}

Autonomous navigation and positioning system,In the field of intelligent robots, robot navigation technology is a new type of technology, its function is crucial, is the core content of intelligent robot research. In order to meet the various needs of the working environment, different paths can be selected when formulating the mobile robot navigation and positioning system, such as using the binocular stereo vision system method and the triangulation principle to explore the movement of the robot in the field and finally determine its location. There are many scientists in China who have analyzed such problems for many years, so they have achieved remarkable results in visual navigation and robot positioning. And mobile robot navigation technology has also made outstanding contributions in industrial undertakings.

The stereo vision system has a certain parallax effect and can complete the target three-dimensional positioning work. However, the reinforced binocular stereo vision system is the best choice and can provide convenience to a large extent. This type of system uses two cameras for reinforcement, which reduces visual differences and is easy to operate. Two cameras as a 
reinforcement, can also be used as a human eyes, using cameras to collect images to restore 3D data information, and ultimately to grasp the specific location of the 3D target. In general, such devices are very demanding, accurate and costly. In addition, it is difficult to grasp the size of the visualization range of the target positioning, and the cumbersome calculation amount is used to calibrate the positions of the two cameras, which increases the error to some extent. Install the mobile terminal actuator in other parts to complete the parallax hand-eye stereo vision system, which can be operated with only one CCD camera.

Hand-eye target positioning system, The hand-eye system is used frequently in the field of automated assembly and aerospace, and this system has accelerated the development of modern industry to some extent. Integrate the vision system into the robot assembly and handling process, and use the assembly parts to identify the installation position of the vision system in the identification process, thereby guiding the robot arm to obtain the accessories to be used, quickly finding the position, and helping to complete the classification, handling and assembly work in industrial production.

\subsection{Computer vision robot navigation}

There are many types of robot navigation technology. The most common ones are map robot navigation, optical flow robot navigation, and geomorphic robot navigation.

Map robot navigation, Based on the predefined map navigation, it can be divided into two types: incremental positioning and absolute positioning. First, when the camera is performing image acquisition, it can quickly extract some features of the image, and establish these features to meet certain relevance, at the same time to build a three-dimensional coordinate system, reasonable control of the remote control in this process. Second, in the sports session, to explore the loop tracking characteristics in the grid. Third, add the active environment network to the map.

Optical flow robot navigation, In recent years, many scholars have successfully developed an optical flow vision system, which is a new type of computer vision system. The optical flow vision system can construct the bee's visual behavior and exercise norms. The advantage of the insect's eyes on both sides is that the characteristics of the movement are formed to guide the bees to behave and not to obtain depth information.

Geomorphic robot navigation, Geomorphic robot navigation is widely used in outdoor environments. The focus of geomorphic robot navigation technology is the identification of digital image patterns. In general, it is the physical color discrimination problem and the physical stripe problem. However, due to the constraints of the environment and the light, the colors of the same essential color of different objects in the environment can be different. Because landform navigation is very difficult to predict in terms of knowledge, it can only complete object processing under the field of view, and cannot construct a map that matches the surrounding environment.

\section{Development trend of computer vision}

Nowadays, many scientists at home and abroad actively explore and analyze the field of machine vision technology, such as the Carnegie Mellon University Robotics Research Institute in the United States and the Autonomous System Center together to create a "3DROOM" system, which is composed of 49 CCD cameras, the main role is to observe the change of dynamic scene, thereby establish a complete set of 3D modeling. The Center for Automatic Control research at the University of Maryland uses a visual motion analysis system to complete the motion capture, modeling, and research tasks of the human body in 3Dscenes, which consist of 64 synchronous cameras. The computer graphics laboratory at Stanford University in the United States used the "LightField" multi-camera array to complete high-performance imaging technology, masked layer reconstruction technology, and high-speed camera technology. The system consists of 128 CMOS cameras. The Mingde College of the United States has proposed a complete standard evaluation system for multi-view 3D reconstruction algorithms, which can accurately quantify and evaluate multi-view 3D reconstruction algorithms, now there are more than 40 multi-viewpoint 
three-dimensional reconstruction algorithm to accurately evaluate the results and rankings. The Hong Kong University of Science and Technology and the Institute of Automation of the Chinese Academy of Sciences and the 3D Visual Computer Robotics Laboratory of Peking University have conducted in-depth research on this work. In addition, the single prism stereo vision system and multi-view stereo vision construction have been further analyzed. In the industrial business, this technology has been used many times and the effect is excellent. Such as drug testing packaging, pharmaceutical printing, printing color testing and so on.

\section{Conclusion}

In summary, with the continuous development and innovation of China's economic construction and science and technology, the development of computer vision technology has been promoted to some extent. Although the technology is still in the development stage, its development trend is very significant. In the later work, computer vision applications will lead China's technology and people to the peak.

\section{References}

[1] Lu Zhiwei, Wang Mingge, Zhao Ruihai. Research and Application of Computer Vision Technology [J]. Digital technology and application, 2016(3):109-110.

[2] Cui Tianyi. Computer vision technology and its application in automation [J]. Computer knowledge and technology, 2016,(12):242-243.

[3] Cao Boyu. Current Status and Prospects of Computer Vision Core Technology [J]. Research, 2016(12):199-120.

[4] Hou Yujing, Sun Fuming. Preliminary Research on Face Detection and Recognition Based on Computer Vision [J]. Computer knowledge and technology, 2017(33):208-209.

[5] Zhang Yuanyuan. Computer vision technology and its application in industry [J]. Newborn, 2017(05):242-243.

[6] Zeng Shuhuai. Research on Urban Construction Height Control Based on Computer Vision Analysis [J]. Construction technology development, 2018(10):20-21. 